\title{
NODAL COSINE SINE MATERIAL INTERPOLATION IN MULTI OBJECTIVE TOPOLOGY OPTIMIZATION WITH THE GLOBAL CRITERIA METHOD FOR LINEAR ELASTO STATIC, HEAT TRANSFER, POTENTIAL FLOW AND BINARY CROSS ENTROPY SHARPENING
}

\author{
Denk, Martin (1); \\ Rother, Klemens (2); \\ Zinßer, Mario (4); \\ Petroll, Christoph (3); \\ Paetzold, Kristin (1) \\ 1: Bundeswehr University Munich, Institute for Technical Product Development; \\ 2: Munich University of Applied Sciences, Institute for Material and Building Research; \\ 3: Bundeswehr University Munich, Institute for Materials, Fuels and Lubricants; \\ 4: Centre
}

\begin{abstract}
Topology optimization is typically used for suitable design suggestions for objectives like mean compliance, mean temperature, or model analysis. Some modern modeling technics in topology optimization require a nodal based material interpolation. Therefore this article is referred to a continuous material interpolation in topology optimization. To cover a smooth and differentiable density field, we address trigonometric shape functions which are infinitely differentiable. Furthermore, we extend a so-known global criteria method with a sharpening function based on binary cross-entropy, so that sharper solutions results. The proposed material interpolation is applied to different applications such as heat transfer, elasto static, and potential flow. Furthermore, these different objectives are together optimized using a multi-objective criterion.
\end{abstract}

Keywords: Lightweight design, Topology Optimisation, Computational design methods, Conceptual design, Design methods

\author{
Contact: \\ Denk, Martin \\ Bundeswehr University Munich \\ Insitute for Technical Product Development \\ Germany \\ martin.denk@unibw.de
}

Cite this article: Denk, M., Rother, K., Zinßer, M., Petroll, C., Paetzold, K. (2021) 'Nodal Cosine Sine Material Interpolation in Multi Objective Topology Optimization with the Global Criteria Method for Linear Elasto Static, Heat Transfer, Potential Flow and Binary Cross Entropy Sharpening', in Proceedings of the International Conference on Engineering Design (ICED21), Gothenburg, Sweden, 16-20 August 2021. DOI:10.1017/pds.2021.486 


\section{INTRODUCTION}

In the industry, problems routinely arise that require making the best possible design decision. Nevertheless, optimization is still underused in the industry. Most modern engineering systems are multidisciplinary, and their analysis is often very complex. Modern design projects with complex problems are often decomposed, and each part is tackled by a multidisciplinary design team (MDT) (Ensici and Badke-Schaub, 2011). Splitting the development into different parts is the simplest decision but comes with a high risk of a suboptimal solution (Song, 2004). The need for multidisciplinary optimization got even bigger when it comes to new manufacturing methods like additive manufacturing (Vantyghem et al., 2018; Yao et al., 2017). The possibility to develop highly customized products accelerates this trend. The result is that multiple disciplines problems occur, which usually interact with each other. A stepwise iteration comes not to reasonable solutions, especially for multiobjective product development. The strongly connected optimization objectives require a nonsequential, parallel approach. When it comes to highly cost-intensive projects like in the field of aerospace engineering, examples already show the usage of solving mechanical loads, vibrations, heat transfer problems, and fluid optimizations together (Bierdel et al., 2017).

The transfer of mechanical loads as well as the dissipation of heat, the transmission of solar power (Gupta et al., 2015), or the reduction of pressure dissipation is required in many applications such as the support and cooling of a battery (Chu et al., 2020), a heat exchanger (Gholamibozanjani and Farid, 2019) or solar power plants. Although these phenomena for various applications can be related and interdependent, most approaches to these problems treat each individual as separate tasks (Chu et al., 2020; Gholamibozanjani and Farid, 2019; Gupta, 2019; Gupta et al., 2015). This lack of a holistic approach can lead to suboptimal solutions. In a battery, for example, the heat exchanging part is typically designed separately from the bearing part sustaining static loads so that only a small amount of heat is dissipated via the respective bearing (Chu et al., 2020). To find suitable design proposals, multiobjective optimization is necessary (Dede, 2009; Kim et al., 2006; Proos et al., 2001), so that for the battery cooperatively, heat dissipation and load sustaining is acquired.

\section{STATE OF THE ART}

The use of topology optimization is applied in many different areas, such as elasto static (Denk et al., 2020; Proos et al., 2001; Zolfagharian et al., 2020b, 2020a), heat conduction (Dede, 2009; Denk et al., 2020; Gersborg-Hansen et al., 2006; Kim et al., 2006; Rodríguez and Pavanello, 2015), fluid mechanics (Dede, 2009), electrostatic (Gupta et al., 2015) or structural dynamics (Kim et al., 2006; Proos et al., 2001). The authors of (Alberto and Sigmund, 2004) summarize several physic types such as electrostatic fields, potential flow, or heat conduction described by the Poisson equation for topology optimization. The optimization of multiple objective functions can be combined using various approaches such as the weighted sum method (Dede, 2009; Denk et al., 2020; Kim et al., 2006; Proos et al., 2001; Rodríguez and Pavanello, 2015), neural networks (Shao et al., 2007), the global criteria method (Denk et al., 2020; Proos et al., 2001) or the normal constraint method (Munk et al., 2018). The different types of physical objectives can be combined, like a) the total pressure energy loss with the mean temperature (Dede, 2009), b) the mean compliance with the mean temperature (Rodríguez and Pavanello, 2015), or c) the mean compliance with the first natural eigenmode (Proos et al., 2001). In addition, to the different types of physical objectives, manufacturing costs and assembly costs can also be taken into account (Guirguis and Aly, 2016) by defining cost functions depending on the shape of the resulting geometry. Stress constraints can be considered (Conlan-Smith and James, 2019; da Silva et al., 2019; Holmberg et al., 2013; Picelli et al., 2018; Yang et al., 2018), which is often covered by choosing a p-norm as a distance functional as well (Conlan-Smith and James, 2019; Holmberg et al., 2013). The ratio weighted sum method similar to (Denk et al., 2020), combines several different objectives by scaling the objective value $\Pi_{j}$ using a full material simulation $\Pi_{j}^{0}$ with

$$
\min \left(\sum_{j=1}^{k} w_{j} \frac{\Pi_{j}}{\Pi_{j}^{0}}\right),
$$

where $w_{j}$ describes the weight, $\Pi_{j}$ the objective of the $j$ th load case. The scaling with $\Pi_{j}^{0}$ is required so that the individual objectives share the same magnitude of, for example, heat dissipation or strain 
energy. The following figure shows the results using two different objectives and different weight factors.

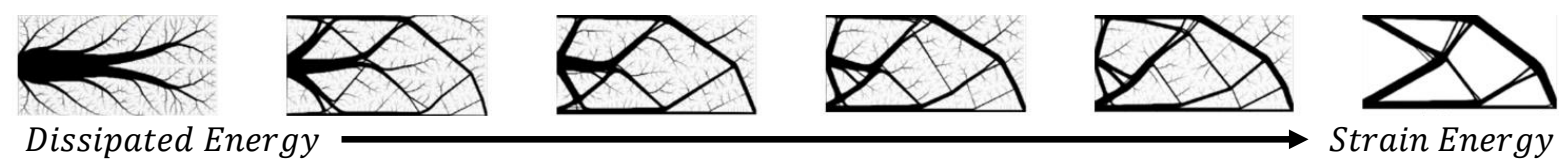

Figure 1: Multiobjective optimization with the ratio weighted sum of (Denk et al., 2020)

Most of the multiobjective optimizations (Dede, 2009; Denk et al., 2020; Kim et al., 2006; Proos et al., 2001; Rodríguez and Pavanello, 2015) are applied on element based material interpolation. So nodebased material interpolation strategies required in (Gupta et al., 2015) cannot be used. We address a node-based interpolation using the global criterion covering multiobjective optimization. To reduce uncertain design variables, we use the classification metric "binary cross-entropy criterion," which should reduce the entropy.

The most common element material interpolation function is the solid isotropic material with penalization (SIMP) based on design variables for elements (Bendsøe and Sigmund, 1999). That approach was extended in changing the interpolation function (Cui et al., 2018; Du et al., 2015) or using a node-based material interpolation field (Guest, 2009; Guest et al., 2004; Kang and Wang, 2012; Rahmatalla and Swan, 2004). So, in contrast to SIMP, the authors of (Cui et al., 2018; Du et al., 2015) chooses a logistic material interpolation function with different parameters applied to the elements. If these interpolation function should be related to composite structures the HashinShtrikman bounds should be stasified (Hashin and Shtrikman, 1963). In our approach we need to violate that criterion similar to (Cui et al., 2018; Du et al., 2015) to provide a interpolation strategy ensuring smooth material distribution in the element. We compare that logistic function and the SIMP method with a trigonometric interpolation strategy. The following figure shows a comparison of SIMP, the logistic functions, and the chosen trigonometric function for different parameters.
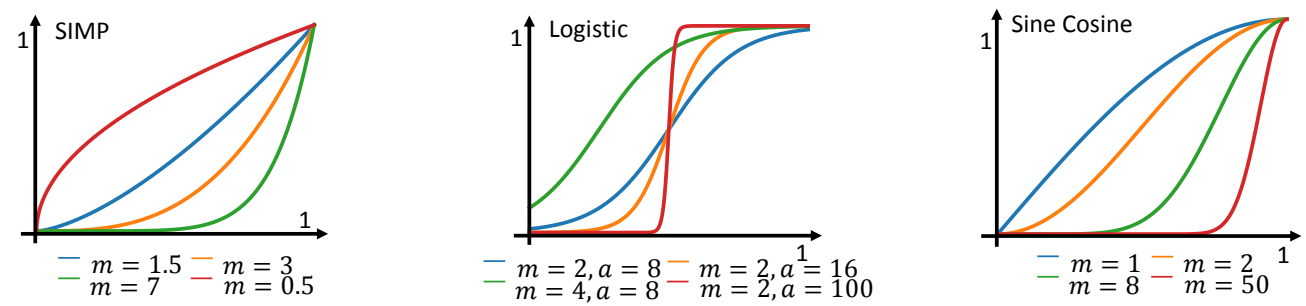

Figure 2: Interpolation functions

Element-based designs can often suffer from numerical instabilities such as one-node connected hinges, mesh dependency, checkboard pattern (Kang and Wang, 2012), which are partly attributed to the constant material density distribution of the local element (Sigmund and Petersson, 1998). The authors of (Matsui and Terada, 2004; Rahmatalla and Swan, 2004) present several options using nodal-based design variables for an element-wise interpolation of the displacement and the design variable field. On that element, the interpolation for the displacements and the design variables can differ (Rahmatalla and Swan, 2004). The authors of (Matsui and Terada, 2004) apply their approach on a three and six-node triangular and a four or eight-node quadrilateral element. The authors of (Rahmatalla and Swan, 2004) summarized several possible configurations for the location of the displacement and design variable nodes so that a Q8/Q4 element represents a quadrilateral element with four design variable nodes and with eight displacement nodes. On that material interpolation phenomena, "islanding" and "layering" structures can occur (Kang and Wang, 2012). In (Paulino and Le, 2009), the authors of (Rahmatalla and Swan, 2004) extended their approach to in-coincident locations of the node-based functions where the interpolation functions overlay several surrounding elements avoiding the "islanding" phenomenon in contrast to (Rahmatalla and Swan, 2004). The shape functions for material and displacement can be independently varied. While (Rahmatalla and Swan, 2004) uses bilinear interpolation functions, the authors of (Kang and Wang, 2012, 2011) use interpolation function derived from Shepard functions of the continuous design variable field to eliminate the "layering" and "islanding" for local and non-local interpolation of the density field. They applied these Shepard interpolation functions in (He et al., 2014) on nonlinear geometric structures. The authors of (Luo et al., 2013) use these Shepard interpolation functions for a dual-level density 
interpolation with meshless field nodes and computational nodes. In a more recent article, topology optimization is applied using isogeometric analysis (IGA) (Dedè et al., 2012; Gao et al., 2020, 2019; Hassani et al., 2013; Liu et al., 2018; Seo et al., 2010). The isogeometric analysis uses basis functions such as NURBS, which are equal for the geometry and the numerical analysis (Nguyen et al., 2015). The authors of (Hassani et al., 2013) use a continuous material distribution function based on NURBS, where the displacement field and density field are processed using NURBS. The authors of (Gao et al., 2019) first use the Shepard function to improve the smoothness of the nodal density, then the NURBS basis functions are combined with that smoothed nodal densities. In contrast to bilinear, NURBS, and Shepard interpolation functions, we use trigonometric shape functions such as sine and cosine for the material interpolation. This shape function ensures continuity and a smooth shape of the material field in one element. Additionally, the material interpolation function has to consider that no negative material properties are resulting in the field (Gao et al., 2019; Kang and Wang, 2012), which is ensured using the proposed trigonometric shape functions. The following figure shows a comparison of the linear, trigonometric, and Shepard interpolation for four elements. The black dots represent full material on the nodes and the with dot zero material.

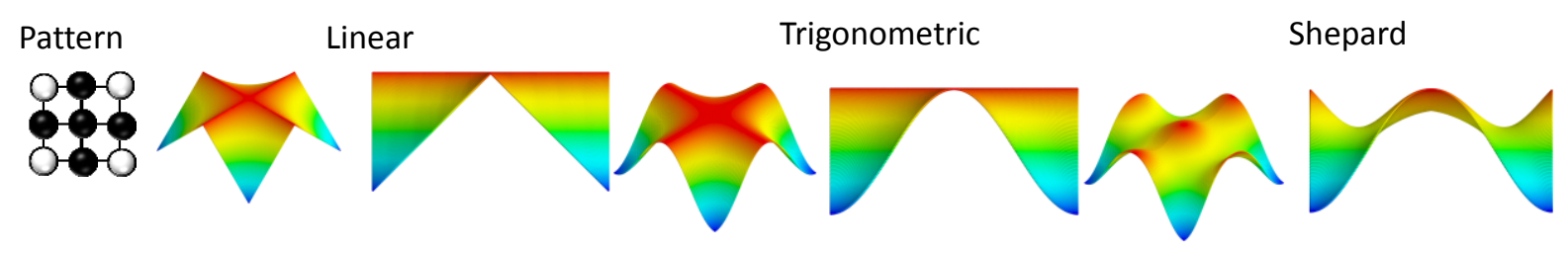

Figure 3: Comparison of the interpolation functions in one element

To summarize our contribution, our research addresses nodal-based material interpolation. We applied the nodal interpolation method on different tasks such as fluid flow, heat transfer, and elasto static by extending the global criteria method proposed in our recent work (Denk et al., 2020). Additionally, we use a new continuous material interpolation based on the cosine/sine function and compare that approach with several material interpolation methods in the literature. As an evaluation, we visually show the results and use a metric based on cross-entropy to address the uncertainty. This evaluation criterion is embedded in the objective function to reduce uncertain design variable decisions (grey areas).

\section{TOPOLOGY OPTIMIZATION WITH CONTINUOUS MATERIAL COSINE SINUE INTERPOLATION AND SHARPNESS METRIC}

To obtain suitable sensitivities, the gradient of the objective function with the design variable must be determined. In topology optimization, the minimization of an objective function $\Pi$ such as the mean compliance, mean temperature, or mean velocity potential (Alberto and Sigmund, 2004) can be selected with

$$
\min \left(\Pi_{j}=\frac{1}{2} \boldsymbol{x}^{T} \boldsymbol{K} \boldsymbol{x}=\frac{1}{2} \sum_{j=1}^{n} \boldsymbol{x}_{u}^{T} \boldsymbol{k}_{u} \boldsymbol{x}_{u}\right),
$$

where $\boldsymbol{K}$ describes the overall stiffness matrix, $\boldsymbol{x}$ the values of the node degrees of freedom such as displacement values, velocity potential or temperature values, $\boldsymbol{k}_{u}$ the element stiffness matrix and und $\boldsymbol{x}_{u}$ the associated displacements on the individual element.

The differentiation of the objective (1) leads to

$$
\frac{\partial \Pi}{\partial \rho_{i}}=\left(\sum_{j=1}^{k} \frac{w_{j}}{\Pi_{j}^{0}} \frac{\partial \Pi_{j}(\boldsymbol{\rho})}{\partial \rho_{i}}\right) .
$$

The design variables $\boldsymbol{\rho}=\left[\rho_{1}, \ldots, \rho_{k}\right]$ are used to change the properties of the stiffness matrix. Differentiating the objective function with the design variables leads to 


$$
\frac{\partial \Pi_{j}}{\partial \rho_{i}}=\frac{1}{2}\left(\frac{\boldsymbol{\partial} \boldsymbol{x}^{T}}{\partial \rho_{i}} \boldsymbol{K} \boldsymbol{x}+\boldsymbol{x}^{T} \frac{\partial \boldsymbol{K}}{\partial \rho_{\boldsymbol{i}}} \boldsymbol{x}+\boldsymbol{x}^{T} \boldsymbol{K} \frac{\partial \boldsymbol{x}}{\partial \rho_{i}}\right)
$$

By using the finite element method, a relationship between the different parts of the equation can be found by differentiating $\boldsymbol{K} \boldsymbol{x}=\boldsymbol{f}$ with

$$
\frac{\partial \boldsymbol{K}}{\partial \rho_{i}} \boldsymbol{x}+\boldsymbol{K} \frac{\partial \boldsymbol{x}}{\partial \rho_{i}}=\frac{\partial \boldsymbol{f}}{\partial \rho_{i}} .
$$

Inserting equation (4) leads to

$$
\frac{\partial \Pi_{j}}{\partial \rho_{i}}=\sum_{r=1}^{k} \boldsymbol{x}_{r, j} \frac{\partial \boldsymbol{f}_{r, j}^{T}}{\partial \rho_{i}}-\frac{1}{2} \sum_{u=1}^{n} \boldsymbol{x}_{u, j}^{T} \frac{\partial \boldsymbol{k}_{u, j}}{\partial \rho_{i}} \boldsymbol{x}_{u, j} .
$$

For our purpose, we don't consider density-dependent loads so that $\frac{\partial \boldsymbol{f}_{r, j}^{T}}{\partial \rho_{i}}=0$.

\subsection{Nodal based material interpolation}

For the material interpolation of the stiffness matrix, we choose the discontinuous density-dependent functional $h\left(\boldsymbol{\rho}_{j}\right)$ and a continuous density field $g\left(\boldsymbol{\rho}_{j}, \Omega\right)$ applied on $\Omega$ with

$$
\boldsymbol{k}_{u}=h\left(\boldsymbol{\rho}_{u}\right) \int_{\Omega} g\left(\boldsymbol{\rho}_{u}, \Omega\right) \boldsymbol{B}^{T} \boldsymbol{D}_{0} \boldsymbol{B} d \Omega
$$

where the derivation leads to

$$
\frac{\partial \boldsymbol{k}_{u}}{\partial \rho_{i}}=\frac{\partial h\left(\boldsymbol{\rho}_{u}\right)}{\partial \rho_{i}} \int_{\Omega} g\left(\boldsymbol{\rho}_{u}, \Omega\right) \boldsymbol{B}^{T} \boldsymbol{D}_{0} \boldsymbol{B} d \Omega+h\left(\boldsymbol{\rho}_{u}\right) \int_{\Omega} \frac{\partial g\left(\boldsymbol{\rho}_{u}, \Omega\right)}{\partial \rho_{i}} \boldsymbol{B}^{T} \boldsymbol{D}_{0} \boldsymbol{B} d \Omega .
$$

Due to the discontinuity of $h\left(\boldsymbol{\rho}_{j}\right)$ only a local element-wise continuous material is resulting. In our work, the density field is selected with

$$
g\left(\boldsymbol{\rho}_{u}, \Omega\right)=\boldsymbol{N} \boldsymbol{\rho}_{u}^{T}=\left[\begin{array}{llll}
N_{1} & N_{2} & N_{3} & N_{4}
\end{array}\right]\left[\begin{array}{llll}
\rho_{1} & \rho_{2} & \rho_{3} & \rho_{4}
\end{array}\right]^{T},
$$

where $\boldsymbol{N}$ describes the $\Omega$ dependent shape function for the material interpolation and $\boldsymbol{\rho}_{j}$ its correspondent design variables and $\rho_{1}, \rho_{2}, \rho_{3}, \rho_{4}$ the design variables referenced to the local element and local shape functions $N_{1}, N_{2}, N_{3}, N_{4}$. As the interpolation function, the constant $N_{c}$, cosine sine $\boldsymbol{N}_{c, s}$ and linear $\boldsymbol{N}_{l}$ dependent shape function is selected with

$$
\boldsymbol{N}_{c}=\left[\begin{array}{c}
0.25 \\
0.25 \\
0.25 \\
0.25
\end{array}\right]^{T} ; \boldsymbol{N}_{c, s}=\left[\begin{array}{l}
\left(\cos \left(x \frac{\pi}{2}\right) \cos \left(y \frac{\pi}{2}\right)\right)^{2} \\
\left(\sin \left(x \frac{\pi}{2}\right) \cos \left(y \frac{\pi}{2}\right)\right)^{2} \\
\left(\sin \left(x \frac{\pi}{2}\right) \sin \left(y \frac{\pi}{2}\right)\right)^{2} \\
\left(\cos \left(x \frac{\pi}{2}\right) \sin \left(y \frac{\pi}{2}\right)\right)^{2}
\end{array}\right]^{2} ; \quad \boldsymbol{N}_{l}=\left[\begin{array}{c}
(1-x)(1-y) \\
x(1-y) \\
x y \\
(1-x) y
\end{array}\right]
$$

The following figure shows the selected interpolation functions for an example in $1 \mathrm{D}$ and $2 \mathrm{D}$. In contrast to the constant shape functions, the linear and the cosine shape function provides a continuous material distribution. Despite the linear function, the trigonometric function is infinitely differentiable, leading to smooth shape representations in the element represented in Figure 3. The chosen domainindependent functions $h\left(\boldsymbol{\rho}_{j}\right)$ are summarized in the following table. 
Table 1: Material interpolation for element and Q4 node-based design variables

\begin{tabular}{|c|c|c|}
\hline SIMP & $h_{\mathrm{sp}}(\rho)=(\rho)^{m}$ & $h_{\mathrm{sp}}\left(\boldsymbol{\rho}_{j}\right)=\left(\frac{1}{4}\left(\rho_{1}+\rho_{2}+\rho_{3}+\rho_{4}\right)\right)^{m}$ \\
\hline Logistic & $h_{\mathrm{lg}}(\rho)=\frac{e^{-\frac{a}{m}+a(\rho)}}{1+e^{-\frac{a}{m}+a(\rho)}}$ & $h_{\mathrm{lg}}\left(\boldsymbol{\rho}_{j}\right)=\frac{e^{-\frac{a}{m}+a \frac{1}{4}\left(\rho_{1}+\rho_{2}+\rho_{3}+\rho_{4}\right)}}{1+e^{-\frac{a}{m}+a \frac{1}{4}\left(\rho_{1}+\rho_{2}+\rho_{3}+\rho_{4}\right)}}$ \\
\hline $\begin{array}{c}\text { Sine } \\
\text { Cosine }\end{array}$ & $h_{\mathrm{sc}}(\rho)=\left(\sin \left(\rho \frac{\pi}{2}\right)\right)^{m}$ & $h_{\mathrm{sc}}\left(\boldsymbol{\rho}_{j}\right)=\left(\sin \left(\frac{1}{4}\left(\rho_{1}+\rho_{2}+\rho_{3}+\rho_{4}\right) \frac{\pi}{2}\right)\right)^{m}$ \\
\hline
\end{tabular}

The following figure shows the interpolation functions for one design variable.

Each node now affects the surrounding elements and its corresponding stiffness matrix so that the influence of the derivation of the sensitivity reduces to the surrounding elements in equation (6). The chosen interpolation functions and the domain-dependent shape functions are variated for comparison. The following table covers fourteen different combinations.

Table 2: Variation of the interpolation function with 14 different combinations

\begin{tabular}{|c|c|c|c|}
\hline 1 & $\boldsymbol{N}_{c}\left[\rho_{1}, \rho_{2}, \rho_{3}, \rho_{4}\right]^{T} h_{\mathrm{sp}}\left(\boldsymbol{\rho}_{j}\right)$ & & $\boldsymbol{N}_{c}\left[h_{\mathrm{lg}}\left(\rho_{1}\right), h_{\mathrm{lg}}\left(\rho_{2}\right), h_{\mathrm{lg}}\left(\rho_{3}\right), h_{\mathrm{lg}}\left(\rho_{4}\right)\right]^{T}$ \\
\hline 2 & $\boldsymbol{N}_{c s}\left[\rho_{1}, \rho_{2}, \rho_{3}, \rho_{4}\right]^{T} h_{\mathrm{sp}}\left(\boldsymbol{\rho}_{j}\right)$ & 9 & $\boldsymbol{N}_{c s}\left[\rho_{1}, \rho_{2}, \rho_{3}, \rho_{4}\right]^{T} h_{\mathrm{lg}}\left(\boldsymbol{\rho}_{j}\right)$ \\
\hline 3 & $\boldsymbol{N}_{l}\left[h_{\mathrm{lg}}\left(\rho_{1}\right), h_{\mathrm{lg}}\left(\rho_{2}\right), h_{\mathrm{lg}}\left(\rho_{3}\right), h_{\mathrm{lg}}\left(\rho_{4}\right)\right]^{T}$ & 10 & $\boldsymbol{N}_{l}\left[\rho_{1}, \rho_{2}, \rho_{3}, \rho_{4}\right]^{T} h_{\mathrm{lg}}\left(\boldsymbol{\rho}_{j}\right)$ \\
\hline 4 & $\boldsymbol{N}_{c s}\left[h_{\mathrm{sp}}\left(\rho_{1}\right), h_{\mathrm{sp}}\left(\rho_{2}\right), h_{\mathrm{sp}}\left(\rho_{3}\right), h_{\mathrm{sp}}\left(\rho_{4}\right)\right]^{T}$ & 11 & $\boldsymbol{N}_{c}\left[h_{\mathrm{sp}}\left(\rho_{1}\right), h_{\mathrm{sp}}\left(\rho_{2}\right), h_{\mathrm{sp}}\left(\rho_{3}\right), h_{\mathrm{sp}}\left(\rho_{4}\right)\right]^{T}$ \\
\hline 5 & $\boldsymbol{N}_{l}\left[\rho_{1}, \rho_{2}, \rho_{3}, \rho_{4}\right]^{T} h_{\mathrm{sp}}\left(\boldsymbol{\rho}_{j}\right)$ & 12 & $\boldsymbol{N}_{c}\left[\rho_{1}, \rho_{2}, \rho_{3}, \rho_{4}\right]^{T} h_{\mathrm{sc}}\left(\boldsymbol{\rho}_{j}\right)$ \\
\hline 6 & $\boldsymbol{N}_{l}\left[h_{\mathrm{sp}}\left(\rho_{1}\right), h_{\mathrm{sp}}\left(\rho_{2}\right), h_{\mathrm{sp}}\left(\rho_{3}\right), h_{\mathrm{sp}}\left(\rho_{4}\right)\right]^{T}$ & 13 & $\boldsymbol{N}_{c s}\left[\rho_{1}, \rho_{2}, \rho_{3}, \rho_{4}\right]^{T} h_{\mathrm{sc}}\left(\boldsymbol{\rho}_{j}\right)$ \\
\hline 7 & $\boldsymbol{N}_{c}\left[\rho_{1}, \rho_{2}, \rho_{3}, \rho_{4}\right]^{T} h_{\mathrm{lg}}\left(\boldsymbol{\rho}_{j}\right)$ & 14 & $\boldsymbol{N}_{l}\left[\rho_{1}, \rho_{2}, \rho_{3}, \rho_{4}\right]^{T} h_{\mathrm{sc}}\left(\boldsymbol{\rho}_{j}\right)$ \\
\hline
\end{tabular}

\subsection{Grey-Scale classification metric for sharpening the results}

In topology optimization, a clear black-white pattern is required (Kang and Wang, 2012) so that the results can be directly interpreted. For binary decisions, the binary cross-entropy loss $m_{c}$ (Denk et al., 2019; Mannor et al., 2005) and polynomial loss function $m_{l}$ with

$$
m_{c}=\frac{1}{n} \sum_{i}-\left(\rho_{i} \log _{\mathrm{a}}\left(\rho_{i}\right)+\left(1-\rho_{i}\right) \log _{\mathrm{a}}\left(1-\rho_{i}\right)\right), \quad m_{l}=\frac{1}{n} \sum_{i}\left(\rho_{i}\left(1-\rho_{i}\right)\right)^{o}
$$

can be chosen with the basis $a$ and the $o$. Both loss functions are minimized if a clear black-white pattern for the design variable is the result. The following figure covers the loss functions with different parameters $a$ and some examples with a high and a small loss value. The maximum value of both loss functions is located by 0.5 so that a high uncertainty results in a high loss value.
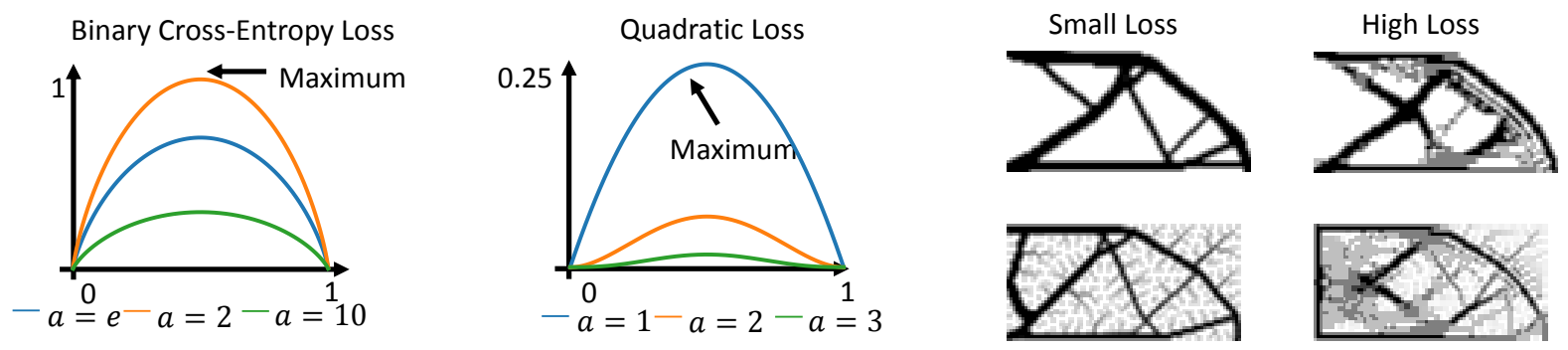
Figure 4: Comparison of the loss function and examples of small and high loss values

With that metric, the global criteria method can be extended by sharpening the design variables with $w_{s} m_{c}$ where the differentiation of the binary cross-entropy $m_{c}$ leads to

$$
\frac{\partial m_{c}}{\partial \rho_{i}}=-1.0 \frac{\left(\ln \left(\rho_{i}\right)-\ln \left(1-\rho_{i}\right)\right)}{\ln (a)} \text {. }
$$

The weight factor $w_{s}$ for the binary cross-entropy can be directly chosen due to the normalization of each objective $\Pi_{j}$ with $\Pi_{j}^{0}$ similar to the weight factors $w_{j}$.

\section{VARIATION OF THE MATERIAL INTERPOLATION STRATEGIES}

In the experimental part, five different cases are investigated. The first three cases are optimized under single optimization criteria. The last two cases consider a) elasto static and heat transfer, and b) elasto static, heat transfer, potential flow with the weight factors of 1.0 for each case. In the first section, the cases are visually compared according to their grey distribution. The second section covers the sharpening effect using the binary cross-entropy as part of the objective function. The following table shows the results of different cases for elasto static, heat transfer, potential flow, and multiobjective cases. Case 2, 4, and 6 results in noise and checkboard parts. Visually the results of 1, 3, 5, 7 are quite similar and converged.

Table 3: Comparison of equations in Table 2 with SIMP $m=3$ and logistic $a=8, m=2$ of

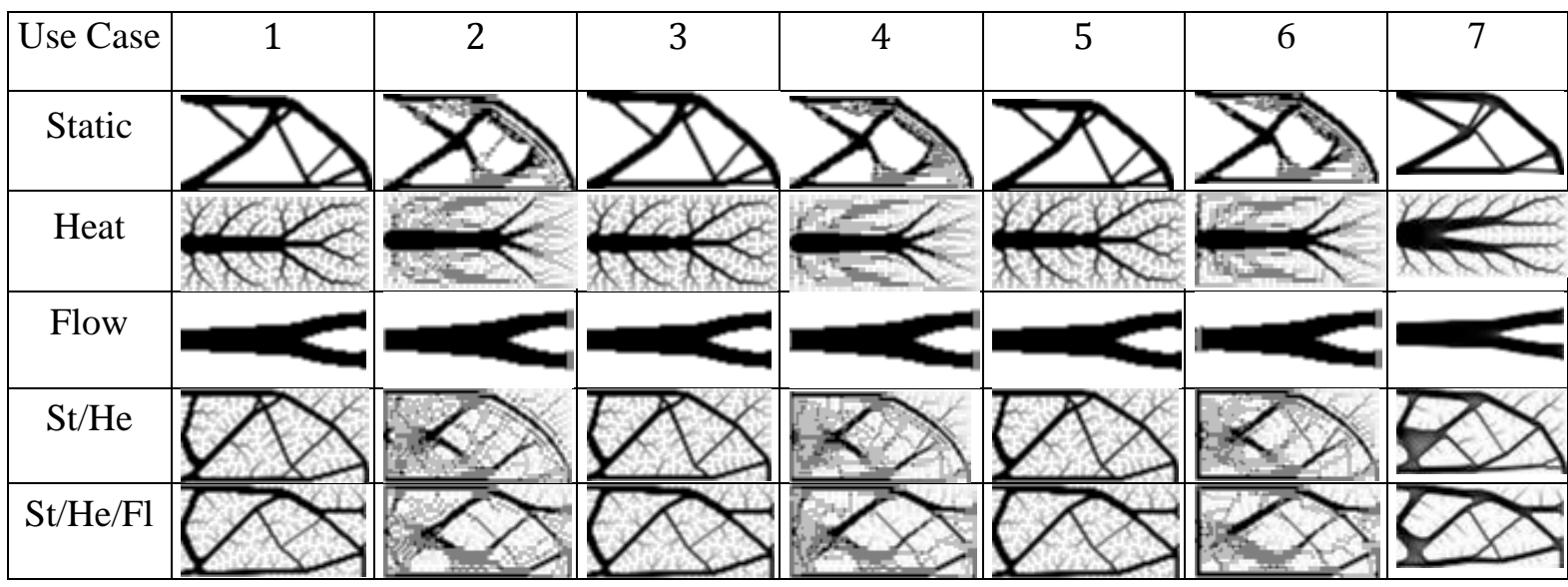

The following table shows the results of different cases for elasto static, heat transfer, potential flow, and multiobjective cases. Case 8 and 11 results in noise and checkboard parts. Especially for the multiobjective optimization, Case 9 and Case 10 shows uncertainty areas.

Table 3: Comparison of interpolation functions with logistic $a=8, m=2$, and sine $m=2$

\begin{tabular}{|c|c|c|c|c|c|c|c|}
\hline Use Case & 8 & 9 & 10 & 11 & 12 & 13 & 14 \\
\hline Static & & & & & & & \\
\hline Heat & & & & & & & \\
\hline Flow & & & & & & & \\
\hline $\mathrm{St} / \mathrm{He}$ & & & & & & & \\
\hline $\mathrm{St} / \mathrm{He} / \mathrm{Fl}$ & & & & & & & \\
\hline
\end{tabular}

The following table covers the results using the sharpening with the cross-entropy. The embedding of the sharpening function in the global criteria method improves the sharpness represented by the binary cross-entropy. The first column shows the result using no sharpening. 
Table 4: Sharpening with the exponent $a=1$ and the basis $a=e$ for the case $13 m=2$

\begin{tabular}{|c|c|c|c|c|c|}
\hline $\begin{array}{l}\text { Use } \\
\text { Case }\end{array}$ & $\begin{aligned} w_{s} & =0.0 \\
a & =2\end{aligned}$ & $\begin{array}{c}w_{s}=1.0 \\
a=2\end{array}$ & $\begin{array}{c}w_{s}=1.0 \\
a=e\end{array}$ & $\begin{array}{c}w_{s}=0.5 \\
a=e\end{array}$ & $\begin{array}{c}w_{s}=0.25 \\
a=e\end{array}$ \\
\hline \multicolumn{6}{|l|}{ Static } \\
\hline \begin{tabular}{l|l}
$m_{c}$ & $m_{l}$ \\
\end{tabular} & \begin{tabular}{l|l|}
0.054 & 0.018 \\
\end{tabular} & 0.053 & \begin{tabular}{l|l}
0.023 & 0.0045 \\
\end{tabular} & \begin{tabular}{l|l}
0.015 & 0.0045 \\
\end{tabular} & \begin{tabular}{l|l}
0.028 & 0.0093 \\
\end{tabular} \\
\hline \multicolumn{6}{|l|}{ Heat } \\
\hline \begin{tabular}{l|l}
$m_{c}$ & $m_{l}$ \\
\end{tabular} & 0.051 & 0.081 & 0.027 & 0.029 & 0.039 \\
\hline \multicolumn{6}{|l|}{ Flow } \\
\hline \begin{tabular}{l|l}
$m_{c}$ & $m_{l}$ \\
\end{tabular} & 0.0082 & 0.061 & 0.0043 & \begin{tabular}{l|l}
0.0060 & 0.00095 \\
\end{tabular} & 0.013 \\
\hline \multicolumn{6}{|l|}{$\mathrm{St} / \mathrm{He}$} \\
\hline \begin{tabular}{l|l}
$m_{c}$ & $m_{l}$ \\
\end{tabular} & 0.051 & 0.097 & 0.035 & 0.039 & 0.14 \\
\hline \multicolumn{6}{|l|}{$\mathrm{St} / \mathrm{He} / \mathrm{Fl}$} \\
\hline$m_{l}$ & 0.047 & 0.035 & 0.038 & 0.040 & 0.14 \\
\hline
\end{tabular}

\section{CONCLUSION AND SUMMARY}

This work addresses the multiobjective topology optimization using a continuous material interpolation, which extends our previous approach presented in (Denk et al., 2020). Despite recent publications, we choose an interpolation shape function method based on trigonometric functions. These interpolation functions result in a smooth material distribution in the element and especially at the boundary of the element. Additionally, we introduce an uncertainty metric based on binary cross-entropy, which is embedded in the multiobjective criterion. With that addition, the binary cross-entropy of the topology optimization results could be reduced, which in consequence results in sharper topology optimization.

The results in Table 3 and Table 4 visually show in examples 2, 4, 6, 8, 11, a lot of checkboard patterns, islands, and grey areas. Therefore, the strategy applying the material interpolation function for each individual node should not proceed. The cases $1,3,5,12,13,14$ show in contrast to 7, 9, 10 quite more details, so that for further proceeding, the material shape functions multiplied by the material interpolation strategy should be applied. The shape functions $g\left(\boldsymbol{\rho}_{u}, \Omega\right)$ for the material interpolation itself has only a small effect on the resulting geometry, whereas the domain-independent function $h\left(\boldsymbol{\rho}_{u}\right)$ changes the resulting shape dramatically. Therefore, the decision of the shape functions applied in $g\left(\boldsymbol{\rho}_{u}, \Omega\right)$ depends more on the desirable properties in consequence, such as the type of continuity and smoothness. Therefore smooth shape functions such as trigonometric shape functions should be applied in further research due to the smooth and infinitely differentiable continuous material interpolation along the element boundaries, which is not possible choosing bilinear and constant shape function and only restricted to finite continuity for NURBS and Shepard functions.

The results of choosing the sharpening metric as part of the objective function shown in Table 5 leads to a sharper black-white configuration. In most use cases, the binary cross-entropy can be reduced by embedding the metric in the objective function with the weight factor of $1.0,0.5$, or 0.25 . Due to the normalization of each objective in the multiobjective ratio global criteria method with $\Pi_{j}^{0}$, the weight factor for the sharpening contribution can be chosen in the same scope as the weight factors (between 0 and 1) of the physical parts $\Pi_{j}$ of the objective. For further investigations, the weight factors for the sharpening metric can consider surrounding design variables so that the influence of the sharpening is affected in a chosen radius. Additionally, the trigonometric shape functions can be chosen as non-local similar to (Kang and Wang, 2011; Paulino and Le, 2009), whereas the shape function itself affects several surrounding elements.

\section{REFERENCES}

Alberto, E., Sigmund, O., 2004. Topology optimization of multiple physics problems modelled by Poisson's equation. Lat. Am. J. Solids Struct. 1, 169-184. 
Bendsøe, M.P., Sigmund, O., 1999. Material interpolation schemes in topology optimization. Arch. Appl. Mech. 69, 635-654. https://doi.org/10.1007/s004190050248

Bierdel, M., Hoschke, K., Pfaff, A., Jäcklein, M., Schimmerohn, M., Wickert, M., 2017. Multidisciplinary Design Optimization of a Satellite Structure by Additive Manufacturing, in: 68th International Astronautical Congress (IAC). Presented at the 68th International Astronautical Congress (IAC), Adelaide, Australia.

Chu, A., Yuan, Y., Zhu, J., Lu, X., Zhou, C., 2020. The Design and Investigation of a Cooling System for a High Power Ni-MH Battery Pack in Hybrid Electric Vehicles. Appl. Sci. 10, 1660. https://doi.org/10.3390/app10051660

Conlan-Smith, C., James, K.A., 2019. A stress-based topology optimization method for heterogeneous structures. Struct. Multidiscip. Optim. 60, 167-183. https://doi.org/10.1007/s00158-019-02207-9

Cui, M., Yang, X., Zhang, Y., Luo, C., Li, G., 2018. An asymptotically concentrated method for structural topology optimization based on the SIMLF interpolation. Int. J. Numer. Methods Eng. 115, 1175-1216. https://doi.org/10.1002/nme.5840

da Silva, G.A., Beck, A.T., Sigmund, O., 2019. Stress-constrained topology optimization considering uniform manufacturing uncertainties. Comput. Methods Appl. Mech. Eng. 344, 512-537. https://doi.org/10.1016/j.cma.2018.10.020

Dede, E., 2009. Multiphysics Topology Optimization of Heat Transfer and Fluid Flow Systems.

Dedè, L., Borden, M.J., Hughes, T.J.R., 2012. Isogeometric Analysis for Topology Optimization with a Phase Field Model. Arch. Comput. Methods Eng. 19, 427-465. https://doi.org/10.1007/s11831-012-9075-z

Denk, M., Paetzold, K., Rother, K., 2019. Feature line detection of noisy triangulated CSGbased objects using deep learning, in: Proceedings of the 30th Symposium Design for X (DFX 2019), DfX. Presented at the DfX Symposium 2019, The Design Society, Jesteburg, Germany, pp. 239-250. https://doi.org/10.35199/dfx2019.21

Denk, M., Rother, K., Paetzold, K., 2020. Multi-Objective Topology Optimization of Heat Conduction and Linear Elastostatic using Weighted Global Criteria Method, in: Proceedings of the 31st Symposium Design for X (DFX2020), DFX. Presented at the DfX Symposium 2020, The Design Society, Bamberg, pp. 91100. https://doi.org/10.35199/dfx2020.10

Du, Y., Yan, S., Zhang, Y., Xie, H., Tian, Q., 2015. A modified interpolation approach for topology optimization. Acta Mech. Solida Sin. 28, 420-430. https://doi.org/10.1016/S0894-9166(15)30027-6

Ensici, A., Badke-Schaub, P., 2011. Information behavior in multidisciplinary design teams, in: Proceedings of the 18th International Conference on Engineering Design (ICED 11), Denmark, pp. 414-423.

Gao, J., Gao, L., Luo, Z., Li, P., 2019. Isogeometric topology optimization for continuum structures using density distribution function. Int. J. Numer. Methods Eng. 119, 991-1017. https://doi.org/10.1002/nme.6081

Gao, J., Luo, Z., Xiao, M., Gao, L., Li, P., 2020. A NURBS-based Multi-Material Interpolation (N-MMI) for isogeometric topology optimization of structures. Appl. Math. Model. 81, 818-843. https://doi.org/10.1016/j.apm.2020.01.006

Gersborg-Hansen, A., Bendsøe, M.P., Sigmund, O., 2006. Topology optimization of heat conduction problems using the finite volume method. Struct. Multidiscip. Optim. 31, 251-259. https://doi.org/10.1007/s00158005-0584-3

Gholamibozanjani, G., Farid, M., 2019. Experimental and mathematical modeling of an air-PCM heat exchanger operating under static and dynamic loads. Energy Build. 202, 109354. https://doi.org/10.1016/j.enbuild.2019.109354

Guest, J.K., 2009. Topology optimization with multiple phase projection. Comput. Methods Appl. Mech. Eng. 199, 123-135. https://doi.org/10.1016/j.cma.2009.09.023

Guest, J.K., Prévost, J.H., Belytschko, T., 2004. Achieving minimum length scale in topology optimization using nodal design variables and projection functions. Int. J. Numer. Methods Eng. 61, 238-254. https://doi.org/10.1002/nme.1064

Guirguis, D., Aly, M.F., 2016. An evolutionary multi-objective topology optimization framework for welded structures, in: 2016 IEEE Congress on Evolutionary Computation (CEC). Presented at the 2016 IEEE Congress on Evolutionary Computation (CEC), pp. 372-378. https://doi.org/10.1109/CEC.2016.7743818

Gupta, D.K., 2019. Topology optimization for high-resolution designs: Application in solar cell metallization. https://doi.org/10.4233/uuid:51dde3f6-2a38-47a0-b719-420ff74ded5d

Gupta, D.K., Langelaar, M., Barink, M., van Keulen, F., 2015. Topology optimization of front metallization patterns for solar cells. Struct. Multidiscip. Optim. 51, 941-955. https://doi.org/10.1007/s00158-014-1185-9

Hashin, Z., Shtrikman, S., 1963. A variational approach to the theory of the elastic behaviour of multiphase materials. J. Mech. Phys. Solids 11, 127-140. https://doi.org/10.1016/0022-5096(63)90060-7

Hassani, B., Tavakkoli, S.M., Ghasemnejad, H., 2013. Simultaneous shape and topology optimization of shell structures. Struct. Multidiscip. Optim. 48, 221-233. https://doi.org/10.1007/s00158-013-0894-9

He, Q., Kang, Z., Wang, Y., 2014. A topology optimization method for geometrically nonlinear structures with meshless analysis and independent density field interpolation. Comput. Mech. 54, 629-644. https://doi.org/10.1007/s00466-014-1011-7 
Holmberg, E., Torstenfelt, B., Klarbring, A., 2013. Stress constrained topology optimization. Struct. Multidiscip. Optim. 48, 33-47. https://doi.org/10.1007/s00158-012-0880-7

Kang, Z., Wang, Y., 2012. A nodal variable method of structural topology optimization based on Shepard interpolant. Int. J. Numer. Methods Eng. 90, 329-342. https://doi.org/10.1002/nme.3321

Kang, Z., Wang, Y., 2011. Structural topology optimization based on non-local Shepard interpolation of density field. Comput. Methods Appl. Mech. Eng. 200, 3515-3525. https://doi.org/10.1016/j.cma.2011.09.001

Kim, W.-Y., Grandhi, R.V., Haney, M., 2006. Multiobjective Evolutionary Structural Optimization Using Combined Static/Dynamic Control Parameters. AIAA J. 44, 794-802. https://doi.org/10.2514/1.16971

Liu, H., Yang, D., Hao, P., Zhu, X., 2018. Isogeometric analysis based topology optimization design with global stress constraint. Comput. Methods Appl. Mech. Eng. 342, 625-652. https://doi.org/10.1016/j.cma.2018.08.013

Luo, Z., Zhang, N., Wang, Y., Gao, W., 2013. Topology optimization of structures using meshless density variable approximants. Int. J. Numer. Methods Eng. 93, 443-464. https://doi.org/10.1002/nme.4394

Mannor, S., Peleg, D., Rubinstein, R., 2005. The cross entropy method for classification, in: Proceedings of the 22nd International Conference on Machine Learning, ICML '05. Association for Computing Machinery, New York, NY, USA, pp. 561-568. https://doi.org/10.1145/1102351.1102422

Matsui, K., Terada, K., 2004. Continuous approximation of material distribution for topology optimization. Int. J. Numer. Methods Eng. 59, 1925-1944. https://doi.org/10.1002/nme.945

Munk, D.J., Kipouros, T., Vio, G.A., Parks, G.T., Steven, G.P., 2018. Multiobjective and multi-physics topology optimization using an updated smart normal constraint bi-directional evolutionary structural optimization method. Struct. Multidiscip. Optim. 57, 665-688. https://doi.org/10.1007/s00158-017-1781-6

Nguyen, V.P., Anitescu, C., Bordas, S.P.A., Rabczuk, T., 2015. Isogeometric analysis: An overview and computer implementation aspects. Math. Comput. Simul. 117, 89-116. https://doi.org/10.1016/j.matcom.2015.05.008

Paulino, G.H., Le, C.H., 2009. A modified Q4/Q4 element for topology optimization. Struct. Multidiscip. Optim. 37, 255-264. https://doi.org/10.1007/s00158-008-0228-5

Picelli, R., Townsend, S., Brampton, C., Norato, J., Kim, H.A., 2018. Stress-based shape and topology optimization with the level set method. Comput. Methods Appl. Mech. Eng. 329, 1-23. https://doi.org/10.1016/j.cma.2017.09.001

Proos, K., Steven, G., Querin, O., Xie, Y., 2001. Multicriterion evolutionary structural optimization using the weighting and the global criterion methods. AIAA J. 39, 2006-2012. https://doi.org/10.2514/3.14961

Rahmatalla, S.F., Swan, C.C., 2004. A Q4/Q4 continuum structural topology optimization implementation. Struct. Multidiscip. Optim. 27, 130-135. https://doi.org/10.1007/s00158-003-0365-9

Rodríguez, S., Pavanello, R., 2015. Thermo-mechanical multi-objective Bidirectional Evolutionary Structural Optimization using weighted sum method for mean compliance and heat conduction problem, in: 11th Argentine Congress on Computational Mechanics (PANACM). Presented at the 11th Argentine Congress on Computational Mechanics (PANACM), Buenos Aires, Argentine.

Seo, Y.-D., Kim, H.-J., Youn, S.-K., 2010. Isogeometric topology optimization using trimmed spline surfaces. Comput. Methods Appl. Mech. Eng. 199, 3270-3296. https://doi.org/10.1016/j.cma.2010.06.033

Shao, X., Chen, Z., Fu, M., Gao, L., 2007. Multi-objective Topology Optimization of Structures Using NN-OC Algorithms*.pp. 204-212. https://doi.org/10.1007/978-3-540-72395-0_26

Sigmund, O., Petersson, J., 1998. Numerical instabilities in topology optimization: A survey on procedures dealing with checkerboards, mesh-dependencies and local minima. Struct. Optim. 16, 68-75. https://doi.org/10.1007/BF01214002

Song, S., 2004. Shared Understanding, Sketching, and Information Seeking and Sharing Behavior in the New Product Design Process. University of California, Berkeley.

Vantyghem, G., Steeman, M., Boel, V., De Corte, W., 2018. Multi-physics topology optimization for 3D-printed structures, in: Proceedings of the IASS Symposium 2018. Presented at the Creativity in Structural Design, Boston, USA.

Yang, D., Liu, H., Zhang, W., Li, S., 2018. Stress-constrained topology optimization based on maximum stress measures. Comput. Struct. 198, 23-39. https://doi.org/10.1016/j.compstruc.2018.01.008

Yao, X., Moon, S.K., Bi, G., 2017. Multidisciplinary design optimization to identify additive manufacturing resources in customized product development. J. Comput. Des. Eng. 4, 131-142. https://doi.org/10.1016/j.jcde.2016.10.001

Zolfagharian, A., Denk, M., Bodaghi, M., Kouzani, A.Z., Kaynak, A., 2020a. Topology-Optimized 4D Printing of a Soft Actuator. Acta Mech. Solida Sin. 33, 418-430. https://doi.org/10.1007/s10338-019-00137-z

Zolfagharian, A., Denk, M., Kouzani, A., Bodaghi, B., Nahavandi, S., Kaynak, A., 2020b. Effects of Topology Optimization in Multimaterial 3D Bioprinting of Soft Actuators. Int. J. Bioprinting 6. https://doi.org/10.18063/ijb.v6i2.260 